\title{
DESEMPEÑO EN MEMORIA DE TRABAJO E INDICADORES COMPORTAMENTALES: RELACIONES ENTRE MEDIDAS DIRECTAS E INDIRECTAS*
}

\author{
RELATIONSHIP BETWEEN PERFORMANCE IN WORKING MEMORY AND BEHAVIORAL \\ INDICATORS OBSERVED IN CLASS
}

\author{
Lorena Canet-Juric**, María Laura Andrés***, Ana García-Coni****, \\ MARÍA M. RICHARD's**** Y DÉBORA BURIN ${ }^{* * * * *}$
}

*Investigación financiada con un subsidio extraordinario del Consejo Nacional de Investigaciones Científicas y Técnicas (CONICET).

${ }^{*}$ Doctora en Psicología y Magister en Psicología Cognitiva y Aprendizaje. Miembro de la Carrera del Investigador Científico del Consejo Nacional de Investigaciones Científicas y Técnicas (CONICET), Docente de Psicología Cognitiva en la Facultad de Psicología y docente del Doctorado en Psicología de la Universidad Nacional de Mar del Plata (UNMP).

E-Mail: Icanetjuric@gmail.com

Universidad Nacional de Mar del Plata (UNMP). Deán Funes 3250 - Cuerpo V - Nivel III (7600) Mar del Plata Buenos Aires - Argentina.

***Doctora en Psicología, Magister en Psicología, Educación y Desarrollo con especificidad en Intervención Psicológica en Contextos de Riesgo y MS en Psicología Cognitiva y Aprendizaje. Becaria Pos-Doctoral del Consejo Nacional de Investigaciones Científicas y Técnicas (CONICET) y Docente en la Cátedra de Psicología Cognitiva en la Facultad de Psicología de la Universidad Nacional de Mar del Plata (UNMP).

****Doctora en Psicología. Miembro de la Carrera del Investigador Científico del Consejo Nacional de Investigaciones Científicas y Técnicas (CONICET) y Docente en la Cátedra Teorías del Aprendizaje de la Facultad de Psicología de la Universidad Nacional de Mar del Plata (UNMP).

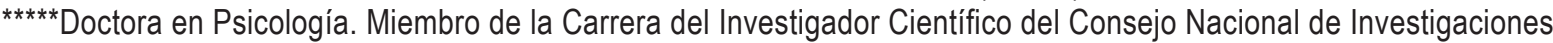
Científicas y Técnicas (CONICET) y Docente en la Universidad Nacional de Mar del Plata (UNMP).

${ }^{* * * * * *}$ Doctora en Psicología. Miembro de la Carrera del Investigador Científico del Consejo Nacional de Investigaciones Científicas y Técnicas (CONICET) y Profesora Adjunta de Psicología Básica en la Universidad Nacional de Buenos Aires (UBA).

Consejo Nacional de Investigaciones Científicas y Técnicas (CONICET) - Instituto de Psicología Básica, Aplicada y Tecnología (IPSIBAT) - Universidad Nacional de Mar del Plata (UNMP).

\section{RESUMEN}

La memoria de trabajo (MT) es un fuerte predictor del desempeño académico por su impacto en la comprensión lectora, la competencia aritmética y los comportamientos necesarios para el aprendizaje escolar. De modo que saber cómo opera la MT en niños de edad escolar y conocer la percepción que los docentes tienen sobre la MT de sus estudiantes es de gran interés. Por eso, los objetivos del trabajo que se informa fueron eva- luar si las observaciones de los docentes sobre las dificultades en la MT de sus alumnos (medidas indirectas) se relacionan con el rendimiento de estos alumnos en pruebas de ejecución de MT (medidas directas) y analizar si estas dificultades percibidas por los docentes se corresponden con la presencia de problemas comportamentales de diversos tipos. Para esto se les administraron a niños de edad escolar pruebas de MT verbal y MT viso-espacial informatizadas, y a sus docentes dos cuestionarios, la Escala Observacional de 
Memoria de Trabajo -WMRS- y la Guía de Observación Comportamental -GOC-. Los resultados obtenidos muestran asociaciones significativas entre indicadores de fallos en MT y la performance de los niños en la tarea de MT verbal, debidas probablemente a que en los comportamientos explorados en la WMRS el componente verbal está más claramente reflejado (e.g., requiere repeticiones de instrucciones). También se encontró que estos fallos se relacionan con problemas comportamentales reportados por los docentes, como ser dificultades atencionales, hiperactividad y autoagresión. Se considera al estudio realizado como una contribución porque no hay escalas observacionales de MT para uso docente, disponibles en nuestro medio.

Palabras clave: Memoria de trabajo; Indicadores comportamentales; Percepción docente; Atención; Hiperactividad.

\section{ABSTRACT}

Working memory $(\mathrm{WM})$ is a strong predictor of academic performance, due to its impact in reading comprehension, arithmetic's competence and the regulated behaviors required for school learning. Thus, knowing about WM operation in schoolaged children and teachers' perception on their students WM is of great value and interest. This is why in the present work we assess whether teachers' reports about their students' WM (indirect measure) is associated with their performance in WM tasks (direct measure), and also to assess whether the difficulties perceived by the teachers correspond to the presence of students' general behavioral issues. For that, we administered computerized verbal WM tasks to school-aged children, in which they had to recall a number of digits (control condition) while saying out loud the color of the items (interference condition), and computerized visual-spatial WM tasks, in which they had to recall the locations of a series of stimuli (control condition) while indicating the color of the stimuli in a color palette (interference condition). We also administered two scales to their teachers: one of them asks about WM specifically (Working Memory Rating Scale) and is composed of 20 short descriptions of behavior problems that allow dis- criminating between children with poor and average WM skills. The teacher rates how typical a behavior is in each child, in a scale that goes from Not typical at all (0) to Very typical (3). The other asks about diverse behavioral problems (Guide of Behavioral Observation) and is composed of the following sub-scales: Physical and Verbal Aggression, Negativism, Transgression, Impulsivity, $\mathrm{Hy}$ peractivity, Attention Deficit and Acceptance. The teacher must choose the option that best describes the behavior in the child in terms of frequency (Never, Sometimes or Often). Our results show significant associations between children performance in the verbal WM task (direct measure) and WM failures' indicators (indirect measures), which can be explained by the fact that the verbal component is more clearly reflected in the behaviors explored in the Working Memory Rating Scale (e.g., loses his place in complicated activities, requires regular repetition of instructions, depends on neighbor to remind them of the current task). We also wanted to look out if there were associations between the WM skills reported by the teachers and the general behavior problems also reported by them, and we found associations between the reported WM difficulties and attention disorders, hyperactivity and self-aggression. This is consistent with the claim that WM and attention deficit in nonclinical population are closely related. With respect to hyperactivity, its relation with WM is not as clear and has not been so frequently reported. With respect to self-aggression, its relation with WM difficulties can be attributed to these children's inability to exercise control over the environment, which leads to frustration and low levels of motivation and self-esteem. Lastly, we found associations between children's performance in the computerized WM tasks and the presence of behavioral problems informed by the teachers. Specifically, proper performance in verbal WM tasks associates with less attention difficulties, where as proper performance in visual-spatial WM tasks associates with being accepted by the group. This last association could be attributed to the fact that many group games require several abilities that involve visual-spatial WM, such as the ability to orientate in space and recall different locations. To conclude, we consider that this work constitutes a contribution because, in the first place, there are no WM observation scales for teachers' use available 
in our environment, and, in the second place, it addresses the need for teachers to acknowledge WM difficulties and consequently be trained for the implementation of effective strategies to improve WM skills in the classroom.

Key words: Working memory; Behavioral indicators; Teacher's perception; Attention; Hyperactivity.

\section{INTRODUCCIÓN}

Muchas de las actividades que se realizan diariamente requieren la habilidad de mantener y manipular información en nuestra mente de manera consciente. El término memoria de trabajo (MT) refiere a los procesos que sostienen y transforman la información online por períodos cortos de tiempo (Baddeley, 2012; Baddeley \& Hitch, 1974; Just \& Carpenter, 1992). Esta capacidad resulta crucial para alcanzar con éxito distintos logros en el curso de la escolaridad.

Adicionalmente, es conocido el papel del coeficiente intelectual en la predicción del éxito académico (Watkins, Lei \& Canivez, 2007); sin embargo, en los últimos años diversos estudios han señalado también a la MT como un predictor relevante del desempeño académico, incluso de mayor importancia que el coeficiente intelectual (CI) (cf., Alloway, T.P. \& Alloway, R.G., 2010; Alloway et al., 2005; Swanson \& Saez, 2003). Por ejemplo, Alloway y colaboradores (2005) observaron que niños con rendimientos promedio o superiores en pruebas de inteligencia presentaban diversos fallos en el ámbito escolar. Seis años después de la primera evaluación se comprobó que su rendimiento en MT predecía en un 95\% su posterior rendimiento escolar. La pregunta es: ¿por qué la MT es un mayor predictor del éxito académico que el CI? Según Alloway y Copello (2013), las tareas de la MT miden algo distinto a lo que mide el CI; mientras que el CI indicaría la presencia de contenidos previamente aprendidos, la MT sería más bien un indicador del potencial de aprendizaje.

El papel predictor de esta habilidad en el éxito académico ha sido comprobado ampliamente (Bull \& Scerif, 2001; de Jong, 1998; Gathercole, Alloway, Willis \& Adams, 2006; Geary, Hoard \& Hamson, 1999; Nation, Adams, Bowyer-Crane \& Snowling, 1999; Stothard \& Hulme, 1992; para una revisión ver Swanson \& Saez, 2003), evidenciándose que las diferencias individuales en MT se relacionan con un amplio rango de habilidades académicas como la lectura, la comprensión lectora y la Matemática.

Así, la MT juega un rol crítico en la lectura, ya que permite a los niños sostener información mientras leen el texto, recuperar conocimiento de la memoria a largo plazo para completar el significado y conservar el sentido de aquello que se leyó a medida que se van leyendo oraciones nuevas. Numerosas investigaciones (Cain, Oakhill \& Bryant, 2004; Canet-Juric, Urquijo, Richard's \& Burin, 2009; Gathercole \& Pickering, 2000; Savage, Lavers \& Pillay, 2007) han demostrado que los niños con dificultades en la lectura suelen mostrar una capacidad limitada de MT.

En cuanto a la competencia matemática, varios estudios (Bull \& Scerif, 2001; Hecht, 2002; Hetcht, Close \& Santisi, 2003; Imbo \& Vandierendonck, 2007; Jordan et al., 2013) encuentran también una fuerte capacidad predictiva de la MT. Por ejemplo, se observó que este sistema de memoria se asocia con la habilidad procedimental de resolución de operaciones aritméticas con números fraccionarios y naturales y predice mejorías en la capacidad de recuperar hechos aritméticos de la memoria a largo plazo (LeFevre et al., 2013). La MT, al permitir la retención de información de manera activa y el procesamiento de los resultados de operaciones parciales en la mente, facilitaría la resolución de problemas aritméticos que involucran diferentes pasos (Cragg \& Gilmore, 2014).

Además de las dificultades académicas, los estudiantes con escasa capacidad de MT exhiben patrones de desajustes conductuales o cognitivos en el contexto escolar. Alloway, 
Gathercole, Kirkwood y Elliott (2009a) exploraron las características cognitivas y comportamentales de niños identificados con déficits en MT. Sus resultados mostraron que los niños con baja amplitud de MT tenían un perfil de comportamientos disruptivos en clase -descriptos por sus docentes- que incluían aspectos como inatención y labilidad atencional, altos niveles de distractibilidad, olvidos recurrentes de las tareas escolares, dificultades en el seguimiento de instrucciones y en la capacidad para completar las tareas escolares. Si bien estos aspectos parecerían coincidir con la dimensión de inatención del Trastorno por Déficit de Atención con o sin Hiperactividad (TDAH - Aronen, Vuontela, Steenari, Salmi \& Carlson, 2005; Martinussen \& Tannock, 2006), estos niños no presentaban los componentes problemáticos de impulsividad e hiperactividad característicos de este trastorno. Distintos estudios han mostrado además que los niños con patrones de comportamientos disruptivos en el contexto escolar poseen menores niveles de rendimiento académico. Por ejemplo, Alatupa y colaboradores (2011) indagaron si los comportamientos disruptivos entendidos como agresividad, hiperactividad y dificultades sociales predecían el desempeño escolar. Para esto, evaluaron a 973 participantes de 3,6 y 9 años de edad, y los siguieron durante su escolaridad obligatoria; es decir, en tercer (6 años), sexto (12 años) y noveno año (15 años) del sistema finlandés. Los resultados indicaron que el comportamiento disruptivo a una edad temprana (3 años) no era predictor del desempeño académico posterior, pero sí lo era en edades más tardías, es decir, a los 6 y 9 años de edad. Esta asociación se mantuvo durante los años posteriores de escolaridad en los que continuaron siendo evaluados.

En síntesis, la evidencia revisada indica que la MT constituye un potente predictor del rendimiento académico al actuar sobre las habilidades académicas de comprensión lectora, competencia matemática y sobre los comportamientos regulados que facilitan el aprendizaje escolar. Debido a esto, resulta de gran valor e interés conocer el funcionamiento de la MT en niños de edad escolar en el contexto áulico y especialmente, indagar acerca de la correspondencia entre el funcionamiento de la MT y las dificultades observadas por los docentes en el aula. Esto permitirá a largo plazo fundamentar la necesidad de capacitaciones alrededor de estas temáticas, en particular sobre herramientas específicas de observación que permitan reconocer déficit de MT (Guzman-Orth et al., 2014). Al respecto, Alloway (2012) evaluó con una entrevista semi-estructurada la capacidad de un grupo de docentes para definir la MT, identificar dificultades de MT entre los estudiantes e implementar estrategias que permitieran ayudar a los estudiantes con estas dificultades. Adicionalmente, se les pidió que identificaran a aquellos alumnos que exhibían algún problema de comportamiento. Los datos indicaron que el conocimiento de los docentes sobre la MT era bastante escaso y que no mencionaban a la misma como causa probable del comportamiento problemático de los niños. Gathercole y colaboradores (2006) argumentan que la explicación para esto es que los docentes suelen pensar que los niños que tienen problemas en su MT son niños faltos de motivación, inatentos o despistados.

Otros estudios han explorado la percepción docente sobre las difícultades de la MT de los estudiantes mediante cuestionarios estructurados. Por ejemplo, Alloway, Gathercole y Kirkwood (2008) diseñaron la Escala Observacional de Memoria de Trabajo (WM RS -Working Memory Rating Scale), que indaga la presencia de comportamientos característicos de niños con dificultades de MT. Los autores encontraron que las puntuaciones en la escala se correspondían con las puntuaciones obtenidas en pruebas ejecutivas de MT. Específicamente, hallaron que la escala correlacionaba altamente con las medidas de MT verbal y en menor medida, aunque de manera significativa, con la MT espacial.

Sin embargo, Guzman-Orth y colaboradores (2014) no hallaron asociaciones entre la percepción docente de las dificultades de MT de estudiantes evaluada mediante la WMRS y medidas de memoria a corto plazo y medidas ejecutivas de MT exclusivamente verbales, aunque sí obtuvieron altas correlaciones 
con indicadores de comportamientos problemáticos en el aula mediante la Escala Abreviada para Docentes de Conners (CTRS-R, 2001). De todos modos, debe señalarse que este estudio fue realizado en niños de habla hispana aprendices del inglés, lo cual podría explicar la falta de asociación entre las puntuaciones de la escala WMRS y las pruebas objetivas de MT, al considerar que estas evaluaciones se realizaron en el contexto del aprendizaje de una nueva lengua. Asimismo, la importancia del desarrollo de los procesos de aprendizaje genera la necesidad de evaluar las habilidades de los niños implicadas en el ámbito educativo con instrumentos válidos, confiables y adaptados a nuestra población escolar (Ison \& Anta, 2006; Monteoliva, Ison \& Pattini, 2014).

Teniendo en cuenta lo anterior, el objetivo general del trabajo realizado consistió en evaluar si las observaciones de los docentes sobre dificultades en la MT de sus alumnos a través de medidas indirectas (como escalas de valoración) se relacionan con el rendimiento de estos en pruebas de ejecución de MT -medidas directas. Adicionalmente se analizó si las dificultades de MT percibidas por los docentes se correspondían con la presencia de problemas comportamentales informados también por los mismos docentes, mediante la administración de la Guía de Observación Comportamental (Ison \& Fachinelli, 1993).

\section{Metodología}

\section{PARTICIPANTES}

La muestra estuvo conformada por 28 niños (18 varones) de primer año de escolaridad primaria básica $(M=6$ años y 8 meses, $D E=3$ meses), pertenecientes a una escuela de gestión privada de la ciudad de Mar del Plata (Prov. de Buenos Aires - Argentina) de nivel socioeconómico medio (Índice de Status Social de Hollingshead; Navarro-Guzmán, 2005) y por los docentes de los niños.

La selección de la muestra fue de tipo no probabilística intencional, según la disponibilidad de la institución educativa. Los crite- rios de inclusión fueron los siguientes: (a) pertenecer al primer año de escolaridad primaria básica, (b) no presentar alteraciones neuropsicológicas y (c) tener un nivel intelectual promedio.

El grupo evaluado asiste a la misma institución educativa desde nivel inicial (en el momento de la evaluación era su cuarto año juntos). Las pruebas se tomaron en el último mes del ciclo lectivo, por lo cual el trabajo de los docentes con el grupo registra un período de un año lectivo, lo que posibilitó un grado de interacción prolongado entre los niños y su docente.

\section{MATERIALES}

Para explorar las dificultades comportamentales vinculadas con la MT de los estudiantes, se utilizó la Escala de Observación de Memoria de Trabajo (Working Memory Rating Scale-WMRS-) de Alloway y colaboradores (2008), luego de ser traducida y adaptada a nuestro idioma. La misma es adecuada para reportar observaciones de niños de 5 a 11 años de edad. Esta escala está conformada por 20 descripciones cortas de problemas de comportamiento que permiten discernir entre niños con escasas habilidades de MT y niños con habilidades promedio. El docente puntúa cuán típico es cada comportamiento de un niño en una escala que va desde Para nada típico (0) a Muy típico (3). Los comportamientos se describen en frases tales como No ofrece respuestas voluntarias en situaciones grupales. Para pasar a la siguiente etapa en una actividad, necesita pistas de parte de los docentes y mezcla el material de manera inapropiada, e.g., combina partes de dos oraciones incorrectamente en vez de leer cada oración con precisión, etc. Este instrumento presenta la ventaja de que permite una evaluación más sistemática de los potenciales problemas de MT que la que se puede llevar a cabo solo mediante observación. Su administración lleva aproximadamente cinco minutos. Se focaliza solo en problemas de MT. Además juega un rol muy importante en familiarizar al docente con situaciones áulicas 
en las cuales esta habilidad falla. La herramienta ha mostrado buenos indicadores internos de confiabilidad y adecuadas propiedades psicométricas como herramienta diagnóstica. También se ha demostrado su validez convergente con medidas tradicionales de MT como el Test AWMA (Automated Working Memory Assessment - Alloway, 2007) y el WISC-IV (Weschler, 2004). La adaptación mostró altos valores de consistencia interna para la muestra analizada en el presente estudio $(\alpha=.97)$.

Para detectar la presencia o ausencia de desajustes conductuales, se aplicó la Guía de Observación Comportamental (GOC) para Niños de Ison y Fachinelli (1993), que informa la clase y frecuencia de conductas problemáticas de niños en edad escolar (más precisamente, de 5 a 13 años de edad - Ison $\&$ Soria, 1997). Se compone de siete subescalas que indagan diferentes tipos de conductas problemáticas.

La Subescala Agresión físico-verbal se refiere a conductas verbales y motoras dirigidas hacia otras personas $u$ objetos físicos con el fin de producir daño y tiene 17 ítems (e.g., "insulta a docentes y / o a compañeros").

La Subescala Negativismo indaga conductas que implican hacer lo contrario de lo que se le ordena al niño o discutir o resistirse a cumplir órdenes, y está compuesta por 3 ítems (e.g., "rechaza órdenes de los adultos").

La Subescala Transgresión refiere a un patrón persistente de conductas en el que el niño viola sistemáticamente los derechos de los demás y normas sociales, está compuesta por 9 ítems (e.g., "trae objetos o dinero que no es de él").

La Sub-escala de Impulsividad se refiere a fallos en la inhibición de la conducta cuando el ambiente lo requiere y a problemas en demorar la satisfacción de una demanda; está integrada por 5 ítems (e.g., "toma decisiones sin pensar las consecuencias").

La Subescala Hiperactividad incluye conductas motoras o verbales de tasa elevada que llaman la atención de las otras personas por ser sostenidas en el tiempo; se compone de 4 ítems (e.g., "se mueve constantemente de un lugar a otro").
La Subescala Déficit de Atención refiere a la dificultad que muestra el niño para mantener focalizada su atención durante períodos cortos de tiempo, obstaculizando el aprendizaje; se compone de 4 ítems (e.g., "alta concentración para el trabajo escolar").

La Subescala Autoagresión refiere a situaciones en las que el daño se dirige hacia el niño mismo; está compuesta por 7 ítems (e.g., "destruye sus producciones").

Por último, la Subescala Aceptabilidad del niño por el grupo de pares refiere justamente a cómo el grupo del niño se relaciona con él y tiene 3 ítems (e.g., "evita acusar a sus pares").

El docente debe indicar la opción que mejor describe la conducta del niño en términos de frecuencia (Nunca, A veces o $A$ menudo), solo teniendo en cuenta las conductas cuya finalidad no sea defenderse a sí mismo o a otros. El análisis de las propiedades psicométricas realizado por los autores del instrumento muestra adecuados niveles de consistencia interna para todas las subescalas excepto para la de Transgresión (Ison \& Fachinelli, 1993). Los coeficientes de consistencia interna obtenidos de la aplicación de las subescalas en nuestra muestra son adecuados, desde .67 hasta .92 para todas las subescalas.

Los docentes de primer año completaron la GOC y la WMRS de cada alumno participante del estudio, quienes realizaron las tareas ejecutivas de MT que se detallan a continuación.

Para evaluar la memoria de trabajo se utilizaron las tareas de evaluación de la MT de la TAC (TEMT-TAC - Canet-Juric, Introzzi, \& Burin, 2015; Introzzi, Canet-Juric, Montes \& Mascarello, 2015), consistentes en adaptaciones informatizadas de las tareas de Fry y Hale (1996) y de Hale, Brownik y Fry (1997). Estas tareas fueron concebidas para evaluar la MT en el ciclo vital y pueden ser usadas a partir de los 6 años de edad. Pueden ser concebidas como una tarea dividida en seis partes: tres corresponden al dominio cognitivo verbal ( $\sin$ interferencia, con interferencia verbal y con interferencia viso-espacial) y tres al dominio cognitivo espacial (sin interferencia, con interferencia viso-espacial y 
con interferencia verbal). Ambos tipos de interferencia tienen como objetivo interrumpir cualquier tipo de estrategia que pudiera facilitar el mantenimiento de la información que se debe recordar. En las tareas sin interferencia se presentaron en una pantalla una serie de ítems de uno en uno (se trató de dígitos en la tarea verbal y de cruces en una matriz de $4 \mathrm{x}$ 4 en la tarea viso-espacial), seguidos de una señal de recordar los ítems recientemente presentados. En las tareas con interferencia verbal los participantes debían decir en voz alta el color en el que aparecía cada ítem. En las tareas con interferencia viso-espacial debían indicar el color de cada ítem señalando en una paleta de colores colocada a la derecha del estímulo presentado. La tarea arroja tres estimaciones de amplitud (cantidad de dígitos o de localizaciones en la matriz que logren ser recordados) para cada dominio (verbal y visoespacial) (ver Canet Juric et al., 2015). Para este trabajo se utilizó un índice abreviado de cada dominio, el dominio verbal por un lado y el viso-espacial por el otro (el índice se constituye por la suma de los resultados parciales de cada una de las tareas del dominio). Esta tarea ha sido utilizada con niños en estudios anteriores y muestra adecuadas propiedades de consistencia interna y validez (e.g., Canet Juric et al., 2015; Hale et al., 1997).

\section{Procedimiento}

Se evaluó a todos los alumnos cuyos padres hubieran prestado su consentimiento por escrito para participar en la investigación. En éste se explicaban los objetivos del estudio y se garantizaba la confidencialidad de la información obtenida y su utilización con fines exclusivamente científicos bajo la Ley Nacional 25.326 de protección de los datos personales. Asimismo, los niños dieron su asentimiento de forma oral. Las tareas de MT de la TAC fueron administradas en una única sesión de evaluación, durante el horario escolar, en una sala de la escuela destinada y acondicionada para tal fin. Paralelamente, se contactó a los docentes de los niños y se les explicó cómo debían llenar ambas escalas.
Transcurrida una semana, se procedió a retirar las evaluaciones de los docentes.

\section{ANÁLISIS DE DATOS}

El plan de análisis estuvo dividido en etapas. En primer lugar, se llevaron a cabo análisis exploratorios a través de pruebas de normalidad e índices de forma (asimetría y curtosis). En segundo lugar, se obtuvieron los estadísticos descriptivos por variables. Posteriormente se realizaron correlaciones entre las dificultades de MT observadas por los docentes, los índices de las tareas ejecutivas de MT y los problemas comportamentales observados por los docentes.

\section{Resultados}

\section{ANÁLISIS DESCRIPTIVOS}

Los estadísticos descriptivos para los distintos índices de desempeño utilizados para la evaluación de la MT y conductas problemáticas se presentan en la Tabla 1. Como se observa en un primer análisis, todas las variables presentan una distribución normal, excepto las escalas comportamentales de Agresión físico-verbal, Déficit de atención y Aceptación por pares, que fueron normalizadas para su análisis posterior.

\section{ANÁLISIS DE CORRELACIONES}

En un segundo análisis se efectuaron correlaciones con el coeficiente $r$ de Pearson entre las dificultades de MT observadas por los docentes, los índices de las tareas ejecutivas de MT y los problemas comportamentales observados por los docentes (ver Tabla 2).

Los resultados mostraron correlaciones estadísticamente significativas entre las dificultades de MT informadas por los docentes y el rendimiento de los niños en tareas de MT verbal $(r=-.43, p<.05)$. Se observó una asociación negativa con el desempeño de los niños en tareas de MT viso-espacial, si bien la 
asociación no alcanzó la significatividad estadística.

En cuanto a las relaciones entre las dificultades de MT informadas por los docentes y los desajustes conductuales también observados por ellos, los resultados mostraron asociaciones con Déficit de Atención $(r=.67$; $p<.01)$ y comportamientos de Autoagresión $(r=.41 ; p<.05)$ e Hiperactividad $(r=.43$; $p<.05)$.

Para establecer con mayor claridad los aspectos de las dificultades en la MT que se asociaban con Déficit de Atención, Autoagresión e Hiperactividad, se realizaron análisis de correlación bivariada entre la Escala WMRS y los ítems de las subescalas Déficit de Atención, Autoagresión e Hiperactividad de la GOC (ver Tabla 3). Como puede observarse, las correlaciones entre las dificultades de MT y los ítems que componen la Subescala Déficit de Atención presentan valores significativos. Con los ítems de la Subescala Autoagresión sólo existe una relación positiva con el ítem referido a Permite que otros lo agredan. Para Hiperactividad se observaron correlaciones con dos de los cuatro ítems.

Además, como puede apreciarse en la Tabla 1 , los resultados mostraron asociaciones entre el desempeño de los niños en tareas de MT verbal y los Déficit de Atención percibidos por los docentes $(r=.49 ; p<.01)$ y la Hiperactividad $(r=-.42 ; p<.05)$ y entre el desempeño de los niños en tareas de MT visoespacial y los comportamientos de Aceptación por pares percibidos por los docentes $(r=.39 ; p<.05)$.

En consecuencia, para conocer en mayor profundidad entre qué aspectos del desempeño en tareas de MT y de los comportamientos se encontraban las asociaciones, se realizaron correlaciones entre el rendimiento en la tarea de ejecución de MT verbal y los ítems que componen las Escalas de Déficit de Atención y de Hiperactividad de la GOC (ver Tabla 4). Se hallaron correlaciones significativas con todos los ítems, menos con el ítem Pérdida de materiales. Para Hiperactividad se observaron correlaciones con todos los ítems menos con el referido a hacer ruidos con objetos sin motivos.
Luego, se realizó un análisis de correlación entre el rendimiento en la tarea de ejecución de MT visoespacial y los ítems que componen la Subescala de Aceptación por pares (ver Tabla 4). En este caso, se halló una correlación significativa con los ítems Posee aceptación social y comparte juegos con su grupo de compañeros y Muestra arrepentimiento si hace algo incorrecto.

\section{Discusión}

La MT refiere al sistema de capacidad limitada encargado del almacenamiento y procesamiento concurrente de estímulos; es decir, se refiere a la capacidad de trabajar con representaciones mentales para el logro de la cognición completa. Como se señaló en la Introducción, se ha demostrado la importancia predictiva de la MT en la comprensión lectora (Cain et al., 2004; Canet-Juric et al., 2009; Savage et al., 2007) y el desempeño matemático (Bull \& Scerif, 2001; Hecht, 2002; Hetcht et al., 2003; Imbo \& Vandierendonck, 2007; Jordan et al., 2013; Mayringer \& Wimmer, 2000), así como en el comportamiento autorregulado en clase (e.g., Alloway et al., 2009a).

El primer objetivo del estudio que se informa consistió en evaluar si los indicadores comportamentales de dificultades en MT informados por los docentes a través de escalas (medidas indirectas) mostraban asociaciones consistentes con el rendimiento de los alumnos en tareas clásicas de ejecución de MT verbal y viso-espacial (medidas directas). Los resultados obtenidos muestran una asociación negativa entre las dificultades de MT percibidas por los docentes y el rendimiento de los alumnos en tareas de MT verbal. En cuanto al rendimiento en tareas de MT viso-espacial, se observó una asociación negativa que no alcanzó la significatividad estadística. Estos resultados están en la misma dirección que los obtenidos por Alloway y colaboradores (2009b), quienes encontraron relaciones moderadas y significativas entre la evaluación de los docentes y el desempeño de los niños en tareas de MT, dando cuenta de que el docen- 
te puede evaluar en cierta manera el rendimiento de MT del niño a través de una observación comportamental, sobre todo en lo referente a los procesamientos verbales, que son los que más se ven reflejados en los comportamientos observables de la escala aplicada (e.g., se olvida de las consignas, pide ayuda permanentemente, no participa de discusiones grupales).

El segundo objetivo fue establecer si las dificultades de MT percibidas por los docentes se correspondían con la presencia de desajustes conductuales informados también por los docentes. Los resultados indican relaciones positivas entre las dificultades de MT y los problemas comportamentales referidos a Atención, Hiperactividad y Autoagresión. En relación con los problemas de atención, estos resultados están en línea con lo planteado por diferentes investigadores (Aronen et al., 2005; Klingber et al., 2005; Martinussen \& Tannock, 2006) que consideran que existen comunalidades entre el comportamiento asociado con déficits en la MT y algunos problemas comportamentales característicos del Trastorno por Déficit de Atención con o sin Hiperactividad (TDAH). En nuestro contexto, un estudio reciente evaluó el desempeño en tareas de MT en niños con desarrollo típico y con TDAH combinado, encontrando que la ejecución de tareas duales de MT, tanto en el domino verbal como viso-espacial, presenta un patrón de desempeño significativamente disminuido en los niños con este trastorno. De hecho, se encontró que estas tareas presentaron índices de sensibilidad y especificidad adecuados para la detección del grupo de pertenencia, clínico o típico (Richard's, Vernucci, Zamora, Canet Juric, Introzzi \& Guardia, en prensa). En general, se han encontrado asociaciones entre la MT y la sintomatología del TDAH referida a la dimensión de inatención, pero no con indicadores de la dimensión de impulsividad. De hecho, en la muestra estudiada es posible observar la ausencia de relaciones entre las dificultades de MT y los indicadores de impulsividad. Estos resultados son consistentes con la hipótesis que sostiene que la MT y el comportamiento deficitario de la atención en población no clínica se en- cuentran estrechamente relacionados. Una posible explicación de esta relación es que tanto los déficit de MT como los de atención ocurrirían debido a que los niños con una capacidad limitada de MT exhiben dificultades para cumplir con las demandas de almacenamiento y procesamiento conjuntos impuesto por las actividades cotidianas que lo muestran como despistado frente a la mirada del observador (ver Gathercole \& Alloway, 2008; Gathercole et al., 2006). La sobrecarga de MT y los denominados efectos abanico consiguientes (tales como sobre-activación de contenidos relevantes sin el borrado de contenidos irrelevantes) conducirían a una pérdida de información importante, comprometiendo la posibilidad del niño de completar distintas tareas y lograr el aprendizaje de un contenido específico. Como consecuencia, el niño perdería su foco o su lugar en la tarea en curso, pareciendo a los ojos del observador inatento o distraido (Gathercole \& Alloway, 2008).

Por otro lado, la relación con la hiperactividad no es tan clara y ha sido reportada en la literatura en menor medida. Sin embargo, algunos autores plantean la posibilidad de que haya relaciones entre la MT y las conductas hiperactivas. Por ejemplo, Klingberg y colaboradores (2005) señalan que luego de una intervención para incrementar la MT, los padres de los niños reportaban disminuciones en síntomas de hiperactividad; sin embargo, estos datos deben tomarse con cautela ya que si bien se presentaba una disminución de estos síntomas, la cantidad de movimientos por minuto de las cabezas de los niños, registrados con una cámara infrarroja, seguía siendo la misma. Adicionalmente, el TDAH se caracteriza por problemas atencionales, excesiva actividad motora y presencia de conductas impulsivas (APA, 2013). Además, es un trastorno que posee alta comorbilidad con diferentes problemas de conducta y emocionales tales como el negativismo y la transgresión (Macià, 2012). En la muestra estudiada no se encontraron relaciones entre la impulsividad, el negativismo y la transgresión y los indicadores comportamentales de fallos en MT o el rendimiento en tareas ejecutivas, por lo que 
nuestros datos abonarían la hipótesis de la especificidad de las dificultades en MT.

En cuanto a las dificultades de MT y los comportamientos de autoagresión, se observó una asociación específicamente entre las dificultades de MT y el ítem de la Subescala Autoagresión referido a que el niño se deja agredir física o verbalmente por otros. Gathercole y Alloway (2008) consideran que si bien los niños que presentan dificultades de MT usualmente tienen relaciones sociales normales con sus pares, suelen mostrarse reservados en actividades grupales y con frecuencia también se observa que no se comprometen en discusiones grupales, particularmente en aquellas que involucran la participación de toda la clase. En estas situaciones, el niño raramente participa, y cuando se requiere su participación activa, puede quedarse en blanco o inmóvil. Por ejemplo, el niño levanta su mano para responder una pregunta del docente, pero cuando el docente le concede el turno, el niño puede mostrarse mudo y volverse incapaz de responder. Estos comportamientos son blanco de críticas y burlas por parte de sus pares, que pueden prolongar la actitud del niño de quietud y/ o falta de respuesta. Sumado a lo anterior, desde el punto de vista de los docentes, los niños con dificultades de MT son también juzgados como niños con baja amplitud atencional y altos niveles de distractibilidad. También son evaluados como niños que se sienten incapaces de ejercer un control sobre su ambiente, lo que les causa frustración, bajos niveles de motivación y posiblemente también bajos niveles de autoestima. Es posible que esto explique las asociaciones observadas entre las dificultades de MT y los comportamientos de autoagresión observados por los docentes.

En tercer lugar, resultados obtenidos mostraron asociaciones entre el rendimiento de los niños en tareas de MT y la presencia de desajustes conductuales observados por los docentes. Por un lado, se observó una relación negativa entre la amplitud de MT verbal en los niños y fallas atencionales informadas por los docentes. Estos resultados se encuentran en línea con los hallazgos de Alloway y colaboradores (2008) en un doble sentido. Primero, el estudio citado informa relaciones entre comportamientos disruptivos característicos de fallos en la MT y la amplitud de la MT. Segundo, estas relaciones se observaron sólo en el caso de la MT verbal; en las tareas de MT espacial los autores no registraron correlaciones significativas, aunque sí se mantuvo la dirección de la relación (a mayor número de indicadores comportamentales, menor amplitud de MT).

Por otro lado, se hallaron relaciones entre la MT viso-espacial de los niños (capacidad involucrada en el procesamiento, codificación, recuerdo y almacenamiento de estímulos tanto de carácter visual como espacial) (Pickering, 2001) y los comportamientos de aceptación de pares informados por los docentes. Específicamente, el análisis de las correlaciones entre la capacidad de MT visoespacial y los diferentes ítems que conforman la Subescala Aceptación por pares mostró una correlación positiva entre la MT visoespacial y los ítems referidos a Posee aceptación social y comparte juegos con su grupo de compañeros y Muestra arrepentimiento si hace algo incorrecto. Muchos de los juegos con pares podrían requerir diversas habilidades que involucran a la MT viso-espacial, como el escaneo de configuraciones espaciales y visuales que permite la orientación espacial hacia un objetivo determinado, la habilidad de orientarse a sí mismo en el espacio, de recuperar diferentes localizaciones simultáneamente y de recordar una secuencia con diferentes localizaciones (Cornoldi \& Vecci, 2003, Mammarella \& Cornoldi, 2005). Ello podría explicar las asociaciones observadas entre la capacidad de los niños de MT viso-espacial y los comportamientos de aceptación de pares registrados por los docentes.

Sumado a lo anterior, si bien la evidencia empírica es escasa, existen diversas hipótesis sobre la implicación de la MT viso-espacial en las interacciones sociales. Estas requieren que el individuo procese información visual como el contacto ocular, las expresiones faciales y las señales idiosincráticas. Se ha encontrado que niños con Síndrome de Asperger que poseen baja capacidad de MT visoespacial, presentan dificultades para procesar 
información visual inherente a las relaciones sociales (Mussarra, 2005).

En síntesis, este estudio ha mostrado asociaciones entre las dificultades de MT percibidas por los docentes y el rendimiento de sus alumnos en tareas de MT verbal y viso-espacial. Específicamente, se observó una asociación negativa entre las dificultades de MT verbal y el rendimiento en tareas de MT verbal. En cuanto a la MT viso-espacial, se observó una asociación negativa, si bien no alcanzó la significatividad estadística.

En segundo lugar, nuestro trabajo permitió mostrar relaciones entre las dificultades de MT y la presencia de problemas comportamentales de los niños percibidos por sus docentes. Particularmente, las dificultades de MT se asociaron con problemas comportamentales de déficit de atención, autoagresión e hiperactividad. Finalmente se registraron asociaciones entre el rendimiento de los niños en tareas de MT y la presencia de problemas comportamentales informados por sus docentes. Específicamente, el buen rendimiento en tareas de MT verbal se asoció con menor presencia de problemas de atención y el buen rendimiento en tareas de MT viso-espacial se asoció con mayor aceptación de pares.

Estos resultados deben ser interpretados con cautela debido a la baja cantidad de participantes en el estudio. Por otro lado, solo fueron los docentes quienes completaron las escalas de dificultades de MT y problemas comportamentales, lo cual puede introducir sesgos en las respuestas. También hay que te- ner en cuenta la particular etapa escolar en que se hallan insertos los niños evaluados; el primer grado es considerado muchas veces como un período de adaptación, los cambios a nivel cognitivo y social que se producen en el niño obligan a tomar con recaudo estos aportes. Futuros estudios podrían incluir comparaciones con otros momentos escolares y más fuentes de información, como por ejemplo cuestionarios o entrevistas con los padres de los niños y / u observaciones directas por parte de otros actores del sistema educativo o evaluadores entrenados en el contexto del aula, teniendo en cuenta no sólo lo observado sino el contexto y las prácticas pedagógicas del mismo docente.

Finalmente, a pesar de estas limitaciones, el trabajo realizado constituye un aporte debido, en primer lugar, a que en la actualidad no se encuentran disponibles en nuestro medio escalas de observación de fallos de MT y en segundo lugar, constituye uno de los primeros aportes en el ámbito hispanohablante para la detección de dificultades en la MT en el aula.

Estos resultados fundamentan la necesidad de concientizar sobre la importancia que tiene para el docente conocer en profundidad este proceso, de manera que pueda acceder a herramientas que le permitan detectar fallos en esta habilidad y posteriormente capacitarse en intervenciones escolares sobre este proceso, para aplicar estrategias pedagógicas efectivas y sencillas para mejorar esta habilidad en el contexto áulico. 
TABLA 1

ESTADÍSTICOS DESCRIPTIVOS PARA LAS DISTINTAS VARIABLES

\begin{tabular}{|l|c|c|c|c|c|}
\hline \multicolumn{1}{|c|}{ Índice } & $M$ & $D E$ & Mínimo & Máximo & Kolmogorov- \\
& & & & \\
& & & & \\
\hline Memoria de Trabajo (MT) & & & & & \\
Dificultades en MT - WMRS & & & & \\
MT Verbal - TAC & 19.45 & 16.68 & 0 & 54 & .08 \\
MT Espacial -TAC & 2.28 & .37 & 1.67 & 3.00 & .21 \\
\hline Guía de Problemas & 2.33 & .51 & 1.33 & 3.67 & .15 \\
Comportamentales (GOC) & & & & & \\
& & & & & \\
Agresión Físico-verbal & .20 & .27 & 0 & 1.06 & .11 \\
$\quad$ Negativismo & .25 & .48 & 0 & 1.67 & .00 \\
$\quad$ Transgresión & .02 & .05 & 0 & .22 & .00 \\
Impulsividad & .30 & .34 & 0 & 1.25 & .08 \\
Déficit de Atención & .56 & .49 & 0 & 1.75 & .38 \\
Autoagresión & .12 & .31 & 0 & 1.71 & .00 \\
Aceptación por pares & .84 & .67 & 0 & 2.00 & .32 \\
Hiperactividad & .48 & .56 & 0 & 1.75 & .20 \\
\hline
\end{tabular}

${ }^{*} p<.05$ 


\begin{tabular}{|c|c|c|c|c|c|c|c|c|c|c|c|c|}
\hline \multirow{12}{*}{ 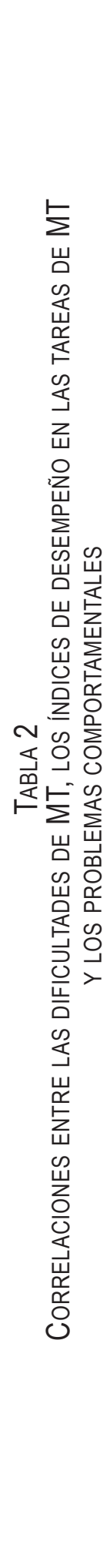 } & 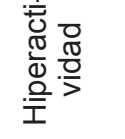 & $\stackrel{*}{\dddot{*}}$ & $\stackrel{*}{\underset{f}{*}}$ & $\mathscr{Q}_{i}$ & $\stackrel{*}{0}$ & $\stackrel{*}{\stackrel{*}{\wedge}}$ & $F$ & 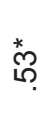 & م & $\stackrel{ }{ }$ & $\stackrel{\infty}{0}$ & - \\
\hline & 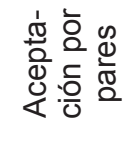 & 농 & ô. & 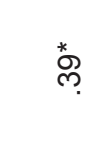 & $\stackrel{2}{\circ}$ & $\tilde{O}_{i}$ & $\stackrel{\nabla}{\leftarrow}$ & ¿̛ & $\stackrel{丶}{\stackrel{9}{*}}$ & ฮั & - & \\
\hline & 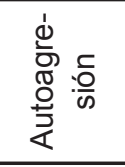 & $\stackrel{*}{\leftarrow}$ & $\overbrace{i}$ & $\stackrel{10}{\circ}$ & $\stackrel{\infty}{o}$ & O̦ $_{i}$ & $\hat{o}_{i}$ & $\stackrel{\Upsilon}{\simeq}$ & $\stackrel{*}{\stackrel{\sim}{\vartheta}}$ & $r$ & & \\
\hline & 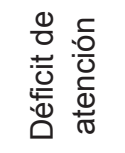 & $\begin{array}{l}\stackrel{*}{*} \\
6 \\
6\end{array}$ & 䓠 & $\stackrel{\oplus}{\oplus}$ & $\stackrel{10}{\leftarrow}$ & $\stackrel{\overbrace{}}{\digamma}$ & 8 & $\stackrel{20}{\leftarrow}$ & - & & & \\
\hline & 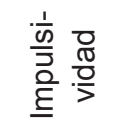 & $\stackrel{\nabla}{r}$ & $\bar{m}$ & $\stackrel{ }{\longleftarrow}$ & $\frac{*}{5}$ & 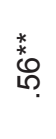 & $\stackrel{p}{N}$ & - & & & & \\
\hline & 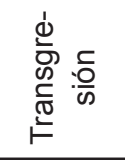 & $\stackrel{10}{ }$ & $\stackrel{\bullet}{\stackrel{n}{*}}$ & $\stackrel{\infty}{\leftarrow}$ & $\underset{i}{\mathbb{N}}$ & $\stackrel{\overbrace{}}{i}$ & - & & & & & \\
\hline & 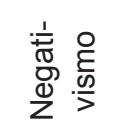 & $\stackrel{\Re}{N}$ & $\stackrel{0}{\stackrel{0}{*}}$ & $\underset{i}{0}$ & $\stackrel{*}{*}$ & - & & & & & & \\
\hline & 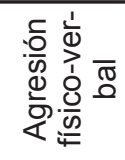 & $\stackrel{ }{\digamma}$ & $\stackrel{\sim}{N}$ & s. & - & & & & & & & \\
\hline & 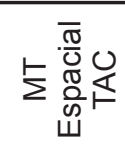 & $\stackrel{\infty}{i}$ & $\stackrel{\Omega}{\longrightarrow}$ & - & & & & & & & & \\
\hline & 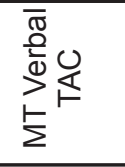 & $\stackrel{*}{\stackrel{*}{9}} \underset{i}{+}$ & $r$ & & & & & & & & & \\
\hline & $\sum_{3}^{\infty}$ & $r$ & & & & & & & & & & \\
\hline & & $\sum_{\substack{\mathcal{Y} \\
\sum}}^{\infty}$ & 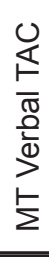 & 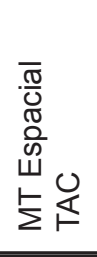 & 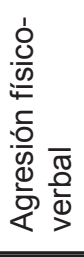 & 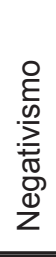 & 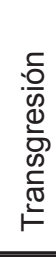 & 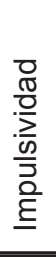 & 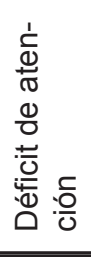 & 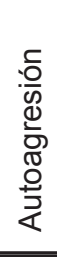 & 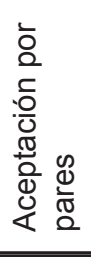 & 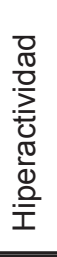 \\
\hline
\end{tabular}




\section{TABLA 3}

CoRRELACIONES ENTRE los ÍTEMS DE LAS SUBESCALAs DÉFICIT DE ATENCIÓN, AUtOAgRESIÓn E HIPERACTIVIDAD DE LA GOC CON LOS PUNTAJES TOTALES DE LA WMRS

\begin{tabular}{|c|c|}
\hline Subescalas e ítems de la GOC & Dificultades en MT (WMRS) \\
\hline \multicolumn{2}{|l|}{ Subescala Déficit de atención } \\
\hline Dificultad en la concentración para el trabajo escolar & $\left..6899^{(*}\right)$ \\
\hline Dificultad en la concentración en tareas extra-escolares y / o familiares & $.671\left(^{* *}\right)$ \\
\hline Dificultad para concentrarse en juegos & $.650(* *)$ \\
\hline Perdida de materiales necesarios para las actividades escolares & $.806(* *)$ \\
\hline \multicolumn{2}{|l|}{ Subescala Autoagresión } \\
\hline Agresión a sí mismo mediante golpes & (a) \\
\hline Presencia de lastimaduras recurrentes & (a) \\
\hline Presencia de cicatrices & (a) \\
\hline Quejas somáticas & .145 \\
\hline Destrucción de sus producciones & (a) \\
\hline Permite que otros lo agredan & $.569\left(^{* *}\right)$ \\
\hline \multicolumn{2}{|l|}{ Subescala Hiperactividad } \\
\hline Se mueve constantemente sin motivo & $.50^{* *}$ \\
\hline Posee dificultad para permanecer sentado & $.48^{* *}$ \\
\hline Habla incesantemente sin motivo & .33 \\
\hline Hace ruidos con objetos sin motivo & .22 \\
\hline
\end{tabular}

** La correlación es significativa al nivel .01 (bilateral).

(a) No se puede calcular porque al menos una variable es constante. 
TABLA 4

CORRELACIONES ENTRE ÍTEMS DE LAS SUBESCALAS DÉFICIT DE ATENCIÓN, HIPERACTIVIDAD Y ACEPTACIÓN POR

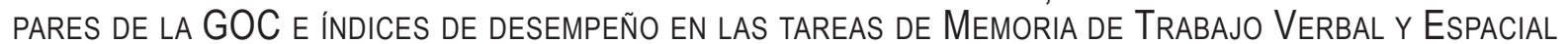
DE LA TAC

\begin{tabular}{|c|c|c|}
\hline Subescalas e ítems & $\begin{array}{c}\text { MT Verbal } \\
\text { TAC }\end{array}$ & $\begin{array}{c}\text { MT Espacial } \\
\text { TAC }\end{array}$ \\
\hline $\begin{array}{l}\text { Subescala Déficit de atención } \\
\text { Dificultad en la concentración para el trabajo escolar } \\
\text { Dificultad en la concentración en tareas extra-escolares y / o familiares } \\
\text { Dificultad para concentrarse en juegos } \\
\text { Pérdida de materiales necesarios para las actividades escolares }\end{array}$ & $\begin{array}{r}-.45^{\star} \\
-.39^{\star} \\
-.47^{\star} \\
.28\end{array}$ & \\
\hline $\begin{array}{l}\text { Subescala Hiperactividad } \\
\text { Se mueve constantemente sin motivo } \\
\text { Posee dificultad para permanecer sentado } \\
\text { Habla incesantemente sin motivo } \\
\text { Hace ruidos con objetos sin motivo }\end{array}$ & $\begin{array}{l}-.38^{*} \\
-.37^{*} \\
-.43^{*} \\
-.36\end{array}$ & \\
\hline $\begin{array}{l}\text { Subescala Aceptación por pares } \\
\text { Posee aceptación social y comparte juegos con su grupo de compañeros } \\
\text { Evita acusar o delatar a sus pares } \\
\text { Muestra arrepentimiento si hace algo incorrecto }\end{array}$ & & $\begin{array}{l}.39^{* *} \\
.21 \\
.36^{*}\end{array}$ \\
\hline
\end{tabular}

${ }^{*} p<.05$

${ }^{* *} p<.01$ 


\section{REFERENCIAS BIBLIOGRÁFICAS}

Alatupa, S., Pulkki-Raback, L., Hintsanen, M., Mullola, S., Lipsanen, J. \& Keltikangas-Karvinen, L. (2011). Childhood disruptive behaviour and school performance across comprehensive school: A prospective cohort study. Psychology, 2(06), 542-551. http://dx.doi.org/10.4236/psy ch.2011.26084

Alloway, T.P. (2007). Automated working memory assessment. London: Pearson Assessment.

Alloway, T.P. (2012). Teachers' perceptions of classroom behaviour and working memory. Educational Research \& Reviews, 7(6), 138142. http://dx.doi.org/10.5897/ERR11.223

Alloway, T.P. \& Alloway, R.G. (2010). Investigating the predictive roles of working memory and IQ in academic attainment. Journal of Experimental Child Psychology, 106, 20-29. http://dx.doi.org/10.1016/j.jecp.2 009.11.003

Alloway, T.P. \& Alloway, R.G. (2013). Working memory across the lifespan : A cross-sectional approach. Journal of Cognitive Psychology, 25(1), 37-41.

Alloway, T.P. \& Copello, E. (2013). Working memory: The what, the why, and the how. The Australian Educational and Developmental Psychologist, 30(02), 105-118. http://dx.doi.or g/10.1017/edp.2013.13

Alloway, T.P., Gathercole, S.E., Adams, A.M., Willis, C., Eaglen, R. \& Lamont, E. (2005). Working memory and other cognitive skills as predictors of progress towards early learning goals at school entry. British Journal of Developmental Psychology, 23, 417-426.

Alloway, T.P., Gathercole, S.E. \& Kirkwood, H.J. (2008). Working Memory Rating Scale. UK: Pearson Education.

Alloway, T.P., Gathercole, S.E., Kirkwood, H. \& Elliott, J. (2009a). The cognitive and behavioral characteristics of children with low working memory. Child Development, 80(2), 606-621. http://dx.doi.org/10.1111/j.1467-8624.2009012 82.x
Alloway, T.P., Gathercole, S.E., Kirkwood, H. \& Elliott, J. (2009b). The working memory rating scale: A classroom-based behavioral assessment of working memory. Learning and Individual Differences, 19(2), 242-245. http://dx.doi.org/ 10.1016/j.lindif.2008.10.003

American Psychiatric Association (2013). Diagnostic and statistical manual of mental disorders (fifth edition). Washington, DC: Author.

Aronen, E.T., Vuontela, V., Steenari, M.R., Salmi, J. \& Carlson, S. (2005). Working memory, psychiatric symptoms, and academic performance at school. Neurobiology of Learning and Memory, 83, 33-42.

Baddeley, A. (2012). Working memory: theories, models, and controversies. Annual Review of Psychology, 63, 1-29. http://dx.doi.org/10.114 6/annurev-psych-120710-100422

Baddeley, A. \& Hitch, G.J. (1974). Working memory. En G.A. Bower (Ed.), The psychology of learning and motivation: Advances in research and theory (pp. 47-89). New York: Academic.

Bull, R. \& Scerif, G. (2001). Executive functioning as a predictor of children's mathematics ability: Inhibition, switching, and working memory. Developmental Neuropsychology, 19(3), 273293. http://dx.doi.org/10.1207/S15326942DN1 9033

Cain, K., Oakhill, J. \& Bryant, P. (2004). Children's reading comprehension ability: Concurrent prediction by working memory, verbal ability and component skills. Journal of Educational Psychology, 96, 31-42. http://dx.doi.org/10.1037/00 22-0663.961.31

Canet-Juric, L., Introzzi, I. \& Burin, D.I. (2015). Desarrollo de la capacidad de memoria de trabajo: Efectos de interferencia inter e intra dominio en niños de edad escolar [Working memory development capacity: Inter- and intradomain interference effects in school age children]. Revista Argentina de Ciencias del Comportamiento, 7(1), 26-37.

Canet-Juric, L., Urquijo, S., Richard's, M.M. \& Burin, D.I. (2009). Predictores cognitivos de 
niveles de comprensión lectora mediante análisis discriminante [Cognitive predictors of reading comprehension levels through discriminant analysis]. International Journal of Psychologycal Research, 2(2), 99-111.

Conners, C.K. (2001). Conners' Rating Scale-Revised: Technical manual. North Tonawanda, NY: Multi- Health Systems.

Cornoldi, C. \& Vecchi, T. (2003). Visuo-spatial working memory and individual differences. New York, NY: Psychology Press.

Cragg, L. \& Gilmore, C. (2014). Skills underlying mathematics: The role of executive function in the development of mathematics proficiency. Trends in Neuroscience and Education, 3, 6368.

de Jong, P.F. (1998). Working memory deficits of reading disabled children. Journal of Experimental Child Psychology, 70, 75-96. http://dx. doi.org/10.1006/jecp.1998.2451

Fry, A.F. \& Hale, S. (1996). Processing speed, working memory, and fluid intelligence: Evidence for a developmental cascade. Psychological Science, 7(4), 237-241.

Gathercole, S.E. \& Alloway, T.P. (2008). Working memory and learning. A practical guide for teachers. Los Angeles: Sage.

Gathercole, S.E., Alloway, T.P., Willis, C. \& Adams, A. (2006). Working memory in children with reading disabilities. Journal of Experimental Child Psychology, 93, 265-281. http:// dx.doi.org/10.1016/j.jecp.2005.08.003

Gathercole, S.E. \& Pickering, S.J. (2000). Working memory deficits in children with low achievement in the national curriculum at 7 year of age. British Journal of Educational Psychology, 70, 177-194. http://dx.doi.org/10.1348/00070990 0158047

Geary, D.C., Hoard, M.K. \& Hamson, C.O. (1999). Numerical and arithmetical cognition: Patterns of functions and deficits in children at risk for a mathematical disability. Journal of Experimental Child Psychology, 74, 213-239. http:// dx.doi.org/10.1006/jecp.1999.2515
Guzman-Orth, D., Grimm, R., Gerber, M., Orosco, M., Swanson, H.L. \& Lussier, C. (2014). Psychometric properties of the working memory rating scale for Spanish-speaking English language learners. Journal of Psychoeducational Assessment, 1-13. http://dx.doi.org/10.1177/07 34282914558710

Hale, S., Bronik, M.D. \& Fry, A. (1997). Verbal and spatial working memory in school-age children: Developmental differences in susceptibility to interference. Developmental Psychology, 33, 364-371. http://dx.doi.org/10.1037/00 1 2-1649.33.2.364

Hecht, S.A. (2002). Counting on working memory in simple arithmetic when counting is used for problem solving. Memory \& Cognition, 30, 447-455. http://dx.doi.org/10.3758/BF031949 45

Hecht, S., Close, L. \& Santisi, M. (2003). Sources of individual differences in fraction skills. Journal of Experimental Child Psychology, 86, 277-302. http://dx.doi.org/10.1016/j.jecp.2003. 08.003

Imbo, I. \& Vandierendonck, A. (2007). The development of strategy use in elementary school children: Working memory and individual differences. Journal of Experimental Child Psychology, 96, 284-309. http://dx.doi.org/10.10 16/j.jecp.2006.09.001

Introzzi, I., Canet-Juric, L., Montes, S., López, S. \& Mascarelo, G. (2015). Procesos inhibitorios y flexibilidad cognitiva : Evidencia a favor de la Teoría de la Inercia Atencional [Inhibitory processes and cognitive flexibility: Evidence for Atentional Inertial Theory]. International Journal of Psychological Research, 8(2), 61-75.

Ison, M.S. \& Anta, F.G. (2006).Estudio normativo del Test de Percepción de Diferencias - CARAS en niños mendocinos [Normative study of the Perception Test of Differences - CA RAS in children from Mendoza]. Interdisciplinaria, 23(2), 203 - 231.

Ison, M.S. \& Fachinelli, C.C. (1993). Guía de Observación Comportamental para Niños [Be- 
havioral Observation Guide for Children]. Interdisciplinaria, 12(1), 11-21.

Ison, M.S. \& Soria, E.R. (1997). Baremo de la Guía de Observación Comportamental para Niños [Scale of the Behavioral Observation Guide for Children]. Interdisciplinaria, 14(1-2), 2546.

Jordan, N.C., Hansen, N., Fuchs, L.S., Siegler, R.S., Gersten, R. \& Micklos, D. (2013). Developmental predictors of fraction concepts and procedures. Journal of Experimental Child Psychology, 116(1), 45-58. http://dx.doi.org/10.1 016/j.jecp.2013.02.001

Just, M. \& Carpenter, P. (1992). A capacity theory of comprehension: Individual differences in working memory. Psychological Review, 99, 122-149. http://dx.doi.org/10.1037/0033-295X. 99.1.122

Klingberg, T., Fernell, E., Olesen, P.J., Johnson, M., Gustafsson, P., Dahlstrom, K. et al. (2005). Computerized training of working memory in children with ADHD. A randomized, controlled trial. Journal of the American Academy of Child and Adolescent Psychiatry, 44, 177-186. http:// dx.doi.org/10.1097/00004583-200502000-000 10

LeFevre, J.A., Berrigan, L., Vendetti, C., Kamawar, D., Bisanz, J., Skwarchuk, S.L. \& Smith-Chant, B.L. (2013). The role of executive attention in the acquisition of mathematical skills for children in Grades 2 through 4. Journal of Experimental Child Psychology, 114(2), 243-261. ht tp://dx.doi.org/10.1016/j.jecp.2012.10.005

Macià, D. (2012). TDAH en la infancia y la adolescencia. Concepto, evaluación y tratamiento [ADHD in childhood and adolescence. Concept, evaluation and treatment]. Madrid: Pirámide.

Mammarella, I.C. \& Cornoldi, C. (2005). Difficulties in the control of irrelevant visuospatial information in children with visuospatial learning disabilities. Acta Psychologica, 118, 221228. ttp://dx.doi.org/10.1016/j.actpsy. 2004.08. 004
Martinussen, R. \& Tannock, R. (2006). Working memory impairments in children with attentiondeficit hyperactivity disorder with and without comorbid language learning disorders. Journal of Clinical and Experimental Neuropsychology, 28, 1073-1094. http://dx.doi.org/10.1080/1380 3390500205700

Mayringer, H., \& Wimmer, H. (2000). Pseudoname learning by German-speaking children with dyslexia: Evidence for a phonological learning deficit. Journal of Experimental Child Psychology, 75, 116-133.

Monteoliva, J.M., Ison, M.S. \& Pattini, A.E. (2014). Evaluación del desempeño atencional en niños: Eficacia, eficiencia y rendimiento [Evaluation of attentional performance in children: Effectiveness, efficiency and performance]. Interdisciplinaria, 31(2), 213-225. https://doi.o $\mathrm{rg} / 1016888$ /interd.2014.31.2.2

Musarra, N.L. (2005). Information-processing skills related to working memory in individuals with Asperger's disorder. Tesis doctoral no publicada. Walden University. Minneapolis, USA.

Nation, K., Adams, J.W., Bowyer-Crane, C.A. \& Snowling, M.J. (1999). Working memory deficits in poor comprehenders reflect underlying language impairments. Journal of Experimental Child Psychology, 73,139-158. http://dx.doi. org/10.1006/jecp.1999.2498

Navarro-Guzmán, J.I. (2005). Índice de status social [Social status index]. Cádiz, España: Universidad de Cádiz.

Pickering, S.J. (2001). The development of visuospatial working memory. Memory (Hove, England), 9(4), 423-432. http://dx.doi.org/10. 1080/09658210143000182

Richard's, M., Vernucci, S., Zamora, E., Canet Juric, L., Introzzi, I. \& Guardia, J. (En prensa). Contribuciones empíricas para la validez de grupos contrastados de la Batería de Tareas de Autorregulación Cognitiva (TAC) [Empirical contributions to the validity of contrasted groups of the Cognitive Self-Regulation Tasks (SRT)]. Interdisciplinaria. 
Savage, R., Lavers, N. \& Pillay, V. (2007). Working memory and reading difficulties: What we know and what we don't know about the relationship. Educational Psychology Review, 19, 185-221. http://dx.doi.org/10.1007/s10648-00 6-9024-1

Stothard, S.E. \& Hulme, C. (1992). Reading comprehension difficulties in children. Reading and Writing: An Interdisciplinary Journal, 4, 245256. http://dx.doi.org/10.1007/BF01027150
Swanson, H.L. \& Saez, L. (2003). Memory difficulties in children and adults with learning disabilities. En H.L. Swanson, S. Graham \& K.R. Harris (Eds.), Handbook of learning disabilities (pp. 182-198). New York: Guildford.

Watkins, M.W., Lei, P.W. \& Canivez, G.L. (2007). Psychometric intelligence and achievement: A cross-lagged panel analysis. Intelligence, 35(1), 59-68.

Wechsler, D. (2004). Wechsler Scale of Intelligence $I V$. London: PearsonAssessment.

Universidad Nacional de Buenos Aires (UBA) Instituto de Psicología Básica, Aplicada y Tecnología (IPSIBAT)

Universidad Nacional de Mar del Plata (UNMP) Consejo Nacional de Investigaciones Cientificas y Técnicas (CONICET) Buenos Aires - República Argentina

Fecha de recepción: 30 de mayo de 2016 Fecha de aceptación: 22 de diciembre de 2016 